\title{
El patrón de lesión y su relación con el índice de gravedad en la baja de combate
}

\author{
García-Cañas R. ${ }^{1 a}$, Navarro-Suay R. ${ }^{2 b}$, Rodríguez-Moro C. ${ }^{3 a}$, Crego-Vita D.M. ${ }^{3 a}$, Bernácer-López J.L. ${ }^{2 a}$, \\ Areta-Jiménez F.J. ${ }^{4 a}$
}

Sanid. mil. 2021; 77 (1): 17-25, ISSN: 1887-8571

\begin{abstract}
RESUMEN
Introducción: Conocer la distribución y el tipo de lesión en el combatiente es una herramienta de utilidad en la planificación y el planeamiento del apoyo sanitario a las operaciones militares. El objetivo de este trabajo es analizar la distribución corporal de las heridas de guerra y determinar la relación con la gravedad según puntuaciones del military Injury Severity Score (mISS) aplicado a bajas de combate. Material y métodos: Se realizó un estudio retrospectivo, descriptivo y transversal sobre todos los pacientes heridos por arma de fuego o explosivos atendidos en el Role 2 español de Herat entre los años 2005 y 2014. Resultados: Se analizaron un total de 911 bajas de combate que presentaron 1.689 lesiones de guerra. La región corporal más afectada fueron los miembros inferiores $(30,19 \%)$, seguido de los miembros superiores $(22,49 \%)$ y la región craneofacial $(19,06 \%)$. Se encontraron diferencias estadísticamente significativas en la distribución de las puntuaciones del mISS en función del número de áreas anatómicas afectadas y entre las bajas que sufrieron lesiones en el tronco y en el abdomen y aquellas que no las sufrieron. Conclusiones: El patrón de lesión en las bajas de combate atendidas en el Role 2 español de Herat estuvo caracterizado por la lesión de las extremidades afectando principalmente a los miembros inferiores. Aquellas bajas con lesiones en el tronco y en el abdomen fueron las que presentaron puntuaciones significativamente más elevadas en el mISS. La gravedad de las bajas estuvo directamente relacionada con el número de áreas anatómicas afectadas.
\end{abstract}

PALABRAS CLAVE: baja de combate, patrón de lesión, índice de gravedad, Role 2, Sanidad Militar, Afganistán.

The injury pattern and its relation to the severity score in the combat casualty

SUMMARY

Introduction: Knowing the distribution and type of injury in the combatant is a useful tool in the medical support planning for military operations. The objective of this work is to analyze the anatomical distribution of war wounds and determine the relationship with severity according to military Injury Severity Score (mISS) applied to combat casualties. Material and methods: A retrospective, descriptive and cross-sectional study was carried out on all patients injured by firearms or explosives treated in the Spanish Role 2 of Herat between 2005 and 2014. Results: A total of 911 combat casualties with 1689 war injuries were analyzed. The body region most affected were the lower limbs (30.19\%), followed by the upper limbs (22.49\%) and the craniofacial region (19.06\%). Statistically significant differences were found in the distribution of the mISS scores according to the number of anatomical areas affected and between casualties that suffered injuries to the trunk and abdomen and those that did not. Conclusions: The injury pattern in combat casualties treated in the Spanish Role 2 of Herat was characterized by injury to the extremities, mainly affecting the lower limbs. Those casualties with injuries to the trunk and abdomen were those with significantly higher scores on the mISS. The severity of the casualties was directly related to the number of anatomical areas affected.

KEYWORDS: Combat casualty, injury pattern, injury severity score, Role 2, Spanish Medical Corps, Afghanistan.

${ }^{1}$ Capitán Médico.

2 Teniente Coronel Médico.

${ }^{3}$ Comandante Médico.

${ }^{4}$ Coronel Médico.

a Servicio de Cirugía Ortopédica y Traumatología. Hospital Central de la Defensa Gómez Ulla, Madrid (España).

b Servicio de Anestesiología, Reanimación y Terapéutica del Dolor. Hospital Central de la Defensa Gómez Ulla, Madrid (España).

Dirección para correspondencia: Rafael García Cañas. Servicio de Cirugía Ortopédica y Traumatología. Hospital Central de la Defensa Gómez Ulla. Glorieta del Ejército 1. 28047 Madrid. España. garciacanas@icloud.com

Recibido: 21 de octubre de 2020

Aceptado: 02 de febrero de 2021

doi: $10.4321 / S 1887-85712021000100003$

\section{INTRODUCCIÓN}

El término de «baja en combate» hace referencia a todas las personas perdidas para el servicio militar, lo que incluye a los muertos en combate, muertos por enfermedad, incapacitados por lesiones físicas o mentales, capturados, desertados y desaparecidos, que ocurren como resultado directo o indirecto de una acción hostil ${ }^{1,2}$. Estas bajas en sí mismas representan un reto para la medicina militar por tratarse habitualmente de pacientes heridos por armas de fuego o explosivos, con lesiones múltiples y complejas, en un entorno hostil, y en un ambiente austero y limitado en recursos humanos y materiales.

Tanto el agente lesivo como el patrón de lesión en las bajas de combate han sufridos cambios constantes a lo largo de tiempo 
debido al desarrollo de nuevos sistemas de armas, de la aparición de nuevos materiales de protección personal y de la propia naturaleza de los conflictos. A su vez, el alcance de las lesiones y la gravedad de las mismas dependerán en gran medida del tipo de agente lesivo y de las medidas de protección pasiva empleadas por la víctima. De modo que las características de las lesiones que encontraremos en el combatiente diferirán ampliamente de las encontradas en la práctica civil en términos de epidemiología, mecanismo de lesión y respuesta fisiopatológica ${ }^{3}$. Es por ello que la estimación de la gravedad en estas bajas de combate constituye un elemento de información y de gran utilidad en la preparación, en la planificación y en el despliegue del apoyo sanitario a las operaciones militares.

El objetivo de este trabajo es analizar la distribución corporal de las heridas de guerra y determinar la relación entre la gravedad y el patrón de lesión mediante el empleo del índice de gravedad military Injury Severity Score (mISS) en las bajas de combate atendidas en el Role 2 español desplegado en Herat (Afganistán).

\section{MATERIAL Y MÉTODOS}

Se plantea un estudio transversal, descriptivo y retrospectivo realizado en el periodo de tiempo comprendido entre los días 1 de mayo de 2005 y el 31 de diciembre de 2014. La población a estudio consistió en todo el personal civil y militar, que hubiese sido atendido en el Role 2 español de Herat tras haber recibido herida por arma de fuego o por artefacto explosivo.

Las variables de estudio fueron sociodemográficas y de control: sexo, edad, militar/civil y ejército de procedencia; independientes: tipo de agente lesivo (arma de fuego, explosivo o arma de fuego más explosivo), área anatómica afectada (cabezacara, cuello, tórax-dorso, abdomen-pelvis, miembros superiores, miembros inferiores), y empleo de medios de protección pasiva (si, no); y dependientes: gravedad de cada lesión según escala military Abbreviated Injury Scale (mAIS) (de 0 a 6), gravedad del paciente según índice mISS (ordinal discontinua, de 1 a 75 excluidos: $7,15,23,28,31,37,39-40,44,46-47,49,52-53$, $55-56,58,60-65$ y 67-74), y gravedad del paciente según puntuaciones agrupadas por niveles de gravedad (menor - puntuaciones: 1-8, moderado - puntuaciones: $9-15$, grave - puntuaciones: 16-24 y crítico - puntuaciones: 25-75).

Los criterios de inclusión fueron: pacientes de ambos sexos, sin límite de edad, personal civil afgano, personal militar afgano, personal militar español, personal militar de ejércitos aliados y personal adscrito al Ministerio de Defensa de España, que hubiesen sufrido una herida por arma de fuego o lesiones por artefacto explosivo tanto sobre el terreno como durante un vuelo. Se eligieron como criterios de exclusión la información errónea o incompleta en las historias clínicas.

Para la realización de este trabajo se han revisado físicamente 32.145 historias clínicas custodiadas y procedentes del archivo de historias clínicas del Role 2 español de Herat, de las que se seleccionaron 923 y fueron excluidas 12 por no presentar el conjunto mínimo de datos requerido.

Se recopilaron los datos en dos hojas de cálculo Excel cumplimentadas de la siguiente manera:
- Una primera tabla Excel donde se realizó una lista numerada con la referencia de la historia clínica manteniendo un algoritmo de cifrado (para cumplir el principio que se recoge en lo establecido en la Ley Orgánica 3/2018; sobre la protección de datos).

- Una segunda tabla Excel, también numerada donde se establecieron las variables completándolas con los datos reflejados directamente desde la historia clínica.

Para el estudio descriptivo de las lesiones se codificó cada lesión según la escala mAIS y según el área anatómica afectada. Para el cálculo del mISS se utilizó el índice de gravedad Injury Severity Score 4 (ISS), cuyo cálculo se realizó mediante la suma del cuadrado de las tres lesiones con puntuación más alta de tres regiones corporales diferentes ${ }^{4}$, aplicado sobre el manual Abbreviated Injury Scale Dictionary versión militar del año $2015^{5}$. Las puntuaciones del mISS fueron agrupadas en cuatro categorías validadas 6 : menor - mild (puntuaciones 1-8), moderado - moderate (9-15), grave - severe (16-24) y crítico - critical (>25), siguiendo los parámetros fijados por el National Trauma Data Bank ${ }^{\circledR}$ del American College of Surgeons o el Japan Trauma Data Bank del Japan Trauma Care and Research.

Este trabajo ha sido aprobado por el Comité de Ética e Investigación Clínica del Hospital Central de la Defensa «Gómez Ulla» (código 36/17) y cuenta con la autorización militar correspondiente.

\section{RESULTADOS}

En un período de estudio de 3.531 días (9 años, 7 meses, y 30 días) se han analizado un total de 911 bajas de combate atendidas en el Role 2 español desplegado en Herat (Afganistán).

La mayoría de las bajas $(96,37 \%)$ eran varones y la edad media de los pacientes fue de 27 años (desviación típica: 8,547). $648(71,13 \%)$ pacientes eran de nacionalidad afgana, de los cuales $459(50,38 \%)$ pertenecían a las fuerzas de seguridad, y 189 pacientes $(20,74 \%)$ eran civiles. $263(28,86 \%)$ bajas de combate correspondieron a militares pertenecientes a la coalición internacional ISAF.

$263(28,87 \%)$ bajas analizadas utilizaron medios de protección pasiva, mientras que $648(71,13 \%)$ no los utilizaron. Aquellas bajas que utilizaron medios de protección pasiva presentaron puntuaciones significativamente más bajas en el mISS.

La mayor parte de las bajas sufrieron lesiones por explosivos $(64,76 \%)$, frente a un $34,68 \%$ que presentaron heridas por de arma de fuego. Las bajas heridas por arma de fuego presentaron puntuaciones más elevadas en el mISS que las bajas heridas por explosivos.

Las 911 bajas de combate estudiadas sufrieron un total de 1.689 lesiones que aumentan a $1.737 \mathrm{si}$ incluimos las lesiones por quemadura. La región corporal más afectada en las bajas analizadas fueron los miembros inferiores $(30,19 \%)$, seguido de los miembros superiores $(22,49 \%)$ y la región craneofacial $(19,06 \%)$ (Figura 1).

En todas las áreas anatómicas estudiadas, el explosivo fue el agente lesivo que provocó el mayor número de lesiones. En 
las regiones de «cabeza-cara» $\mathrm{y}$ «cuello» los explosivos motivaron en torno al 90\% de las lesiones registradas (Tabla 1). En el grupo de bajas heridas por explosivos, las «extremidades inferiores» $(27,4 \%)$ fueron la región corporal más afectada, seguido por la «cabeza-cara» $(22,99 \%)$ y las «extremidades superiores» $(21,15 \%)$. Mientras que en el grupo de bajas heridas por arma de fuego, las «extremidades inferiores» fueron la región corporal más afectada, seguido por las «extremidades superio- res» $(25,87 \%)$ y la región «tórax-dorso» $(13,15 \%)$ (Tabla 2$)$. En ambos grupos, las extremidades fueron la región corporal más frecuentemente afectada por heridas de guerra.

La distribución de las puntuaciones del mISS fue significativamente mayor en aquellas bajas con lesiones en tórax/dorso, abdomen y miembros superiores, respecto a las bajas que no presentaron lesiones en el tórax/dorso y en los miembros superiores, respectivamente (Tabla 3 ).

Tabla 1. Distribución de lesiones por el tipo de agente lesivo en función de la región corporal afectada.

\begin{tabular}{|c|c|c|c|c|c|c|c|}
\hline \multirow{2}{*}{ REGIÓN CORPORAL } & \multirow{2}{*}{$\mathbf{N}$} & \multicolumn{2}{|c|}{ EXPLOSIVOS } & \multicolumn{2}{|c|}{ ARMA DE FUEGO } & \multicolumn{2}{|c|}{ EXPLOSIVO + ARMA DE FUEGO } \\
\hline & & $\mathbf{N}$ & $\%$ & $\mathbf{N}$ & $\%$ & $\mathbf{N}$ & $\%$ \\
\hline Cabeza-Cara & 322 & 287 & 89,13 & 34 & 10,55 & 1 & 0,31 \\
\hline Cuello & 111 & 101 & 90,99 & 10 & 9 & 0 & 0 \\
\hline Tórax-Dorso & 201 & 141 & 70,14 & 58 & 28,85 & 2 & 0,99 \\
\hline Abdomen-Pelvis & 165 & 113 & 68,48 & 51 & 30,9 & 1 & 0,6 \\
\hline Extremidades superiores & 380 & 264 & 75,42 & 111 & 29,21 & 5 & 1,31 \\
\hline Extremidades inferiores & 510 & 342 & 67,05 & 165 & 32,35 & 3 & 0,58 \\
\hline TOTAL & 1689 & 1248 & 73,88 & 429 & 25,39 & 12 & 0,71 \\
\hline
\end{tabular}

Fuente: Elaboración propia.

Tabla 2. Distribución de lesiones según región corporal afectada en función del agente lesivo.

\begin{tabular}{|l|c|c|c|c|c|c|c|c|}
\hline \multicolumn{2}{|c|}{ AGENTE LESIVO } & \multicolumn{9}{c|}{ REGIÓN CORPORAL } \\
\cline { 3 - 9 } \multicolumn{2}{|c|}{} & $\begin{array}{c}\text { Cabeza } \\
\text { Cara }\end{array}$ & Cuello & $\begin{array}{c}\text { Tórax } \\
\text { Dorso }\end{array}$ & $\begin{array}{c}\text { Abdomen } \\
\text { Pelvis }\end{array}$ & $\begin{array}{c}\text { Extremidades } \\
\text { superiores }\end{array}$ & $\begin{array}{c}\text { Extremidades } \\
\text { inferiores }\end{array}$ & $\begin{array}{c}\text { TOTAL } \\
\text { Explosivos }\end{array}$ \\
& $\mathrm{N}$ & 287 & 101 & 141 & 113 & 264 & 342 \\
& $\%$ & 22,99 & 8,09 & 11,29 & 9,05 & 21,15 & 1248 \\
Arma de fuego & $\mathrm{N}$ & 34 & 10 & 58 & 51 & 111 & 100 \\
\hline \multirow{2}{*}{ Explosivos + Arma de fuego } & $\%$ & 7,92 & 2,33 & 13,51 & 11,88 & 25,87 & 38,46 & 100 \\
\hline
\end{tabular}

Fuente: Elaboración propia.

Tabla 3. Asociación entre la puntuación del mISS y el patrón lesional.

\begin{tabular}{|c|c|c|c|c|c|c|c|c|c|c|c|}
\hline \multirow{2}{*}{$\begin{array}{l}\text { PATRÓN LESIONAL: ÁREA } \\
\text { ANATÓMICA/QUEMADO }\end{array}$} & \multicolumn{11}{|c|}{ PUNTUACIÓN DEL mISS } \\
\hline & Afectado & $\mathbf{N}$ & $\%$ & Media & DE & Mediana & $\mathbf{P 2 5}$ & P75 & Mínimo & Máximo & $\mathbf{p}^{*}$ \\
\hline \multirow{2}{*}{ Cabeza/cara } & No & 589 & 64,65 & 10,88 & 10,29 & 8 & 4 & 16 & 1 & 57 & \multirow{2}{*}{0,175} \\
\hline & Sí & 322 & 35,35 & 12,52 & 12,08 & 8 & 4 & 19 & 1 & 57 & \\
\hline \multirow{2}{*}{ Cuello } & No & 800 & 87,82 & 11,47 & 10,72 & 8 & 4 & 16 & 1 & 57 & \multirow{2}{*}{0,093} \\
\hline & Sí & 111 & 12,18 & 11,41 & 12,75 & 5 & 2 & 17 & 1 & 57 & \\
\hline \multirow{2}{*}{ Tórax/dorso } & No & 710 & 77,94 & 9,35 & 8,70 & 5 & 4 & 13 & 1 & 50 & \multirow{2}{*}{0,000} \\
\hline & Sí & 201 & 22,06 & 18,93 & 14,41 & 17 & 6 & 27 & 1 & 57 & \\
\hline \multirow{2}{*}{ Abdomen } & No & 746 & 81,89 & 9,50 & 9,48 & 5 & 4 & 13 & 1 & 52 & \multirow{2}{*}{0,000} \\
\hline & Sí & 165 & 18,11 & 20,34 & 12,77 & 19 & 10 & 27 & 1 & 57 & \\
\hline \multirow{2}{*}{ Miembros superiores } & No & 531 & 58,29 & 11,04 & 10,93 & 6 & 4 & 16 & 1 & 57 & \multirow{2}{*}{0,007} \\
\hline & Sí & 380 & 41,71 & 12,05 & 11,03 & 8 & 4 & 17 & 1 & 52 & \\
\hline \multirow{2}{*}{ Miembros inferiores } & No & 401 & 44,02 & 11,38 & 11,29 & 8 & 4 & 16 & 1 & 57 & \multirow{2}{*}{0,149} \\
\hline & Sí & 510 & 55,98 & 11,52 & 10,73 & 8 & 4 & 16 & 1 & 57 & \\
\hline \multirow{2}{*}{ Quemado } & No & 863 & 94,73 & 11,27 & 10,79 & 8 & 4 & 16 & 1 & 57 & \multirow{2}{*}{0,086} \\
\hline & Sí & 48 & 5,27 & 14,94 & 13,52 & 8 & 5 & 24 & 1 & 48 & \\
\hline \multicolumn{2}{|l|}{ Total } & 911 & 100 & 11,46 & 10,98 & 8 & 4 & 16 & 1 & 57 & \\
\hline
\end{tabular}

N: Número de pacientes. DE: Desviación estándar. P25: Percentil 25. P75: Percentil 75. *U de Mann-Whitney.

Fuente: Elaboración propia. 


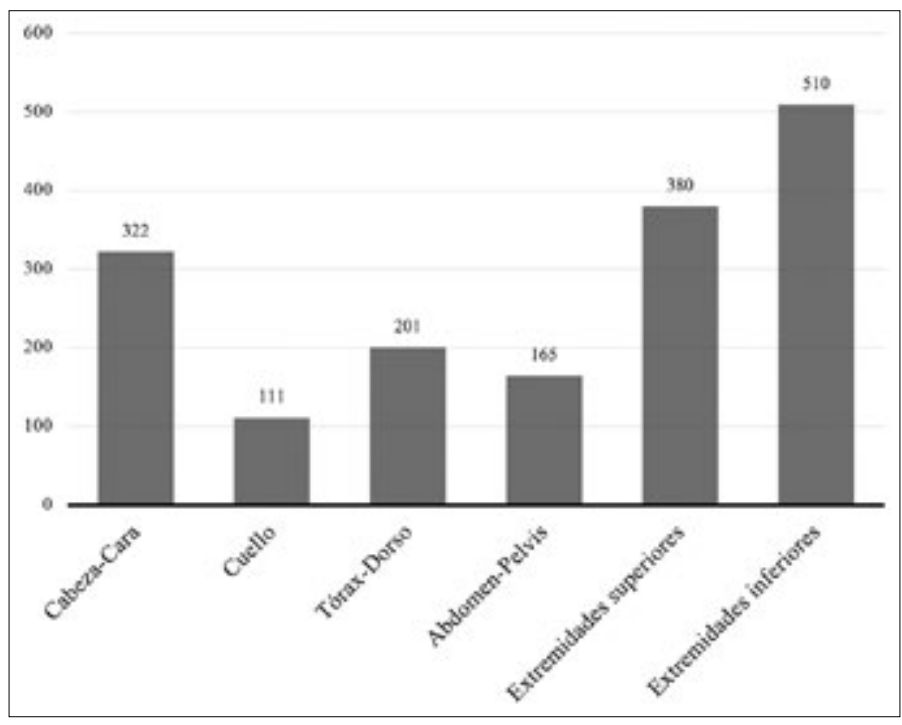

Figura 1. Distribución anatómica de las heridas de guerra.
En el análisis de asociaciones entre los niveles de gravedad del mISS y el patrón de lesión, las bajas que presentaron lesiones en la cabeza/cara, en el cuello, en el tórax/dorso y en el abdomen presentaron puntuaciones significativamente más elevadas que aquellas bajas que no sufrieron lesiones en la cabeza/cara, en el cuello, en el tórax/dorso y en el abdomen, respectivamente (Tabla 4).

La distribución de las puntuaciones del mISS en función del número de áreas anatómicas afectadas mostró diferencias estadísticamente significativas $(\mathrm{p}=0,000)$. Por ejemplo, la puntuación del mISS en los lesionados en una sola área anatómica (mediana: 5; RIQ: 4-10) fue menor que en los lesionados en 6 áreas anatómicas (mediana: 41; RIQ: 6-48) (Tabla 5). Por otro lado, fueron clasificados como nivel de gravedad crítico 34 (7,71\%) heridos sólo en un área anatómica, y cinco $(38,46 \%)$ heridos en 5 áreas anatómicas, a la vez que fueron clasificados como nivel de gravedad menor $264(59,86 \%)$ heridos sólo en un área anatómica, y $12(23,53 \%)$ heridos en 4 áreas anatómicas (Tabla 6).

Tabla 4. Asociación entre el nivel de gravedad del mISS y el patrón lesional.

\begin{tabular}{|c|c|c|c|c|c|c|c|c|}
\hline \multirow{2}{*}{$\begin{array}{c}\text { PATRÓN LESIONAL: ÁREA } \\
\text { ANATÓMICA/QUEMADO }\end{array}$} & \multicolumn{8}{|c|}{ NIVEL DE GRAVEDAD mISS } \\
\hline & \multicolumn{2}{|c|}{ Afectado } & \multirow{3}{*}{$\begin{array}{c}\text { Menor } \\
311 \\
52,80 \\
\end{array}$} & \multirow{3}{*}{$\begin{array}{c}\text { Moderado } \\
119 \\
20,20 \\
\end{array}$} & \multirow{3}{*}{$\begin{array}{c}\text { Grave } \\
86 \\
14,60 \\
\end{array}$} & \multirow{3}{*}{$\begin{array}{c}\text { Crítico } \\
73 \\
12,39 \\
\end{array}$} & \multirow{3}{*}{\begin{tabular}{c|} 
Total \\
589 \\
100 \\
\end{tabular}} & $\mathbf{p}^{*}$ \\
\hline \multirow{4}{*}{ Cabeza/cara } & \multirow{2}{*}{ No } & $\mathrm{N}$ & & & & & & \multirow{4}{*}{0,025} \\
\hline & & $\%$ & & & & & & \\
\hline & \multirow{2}{*}{ Sí } & $\mathrm{N}$ & 169 & 48 & 44 & 61 & 322 & \\
\hline & & $\%$ & 52,48 & 14,91 & 13,66 & 18,94 & 100 & \\
\hline \multirow{4}{*}{ Cuello } & \multirow{2}{*}{ No } & $\mathrm{N}$ & 412 & 154 & 123 & 111 & 800 & \multirow{4}{*}{0,004} \\
\hline & & $\%$ & 51,50 & 19,25 & 15,38 & 13,88 & 100 & \\
\hline & \multirow{2}{*}{ Sí } & $\mathrm{N}$ & 68 & 13 & 7 & 23 & 111 & \\
\hline & & $\%$ & 61,26 & 11,71 & 6,31 & 20,72 & 100 & \\
\hline \multirow{4}{*}{ Tórax/dorso } & \multirow{2}{*}{ No } & $\mathrm{N}$ & 417 & 134 & 91 & 68 & 710 & \multirow{4}{*}{0,000} \\
\hline & & $\%$ & 58,73 & 18,87 & 12,82 & 9,58 & 100 & \\
\hline & \multirow{2}{*}{ Sí } & $\mathrm{N}$ & 63 & 33 & 39 & 66 & 201 & \\
\hline & & $\%$ & 31,34 & 16,42 & 19,40 & 32,84 & 100 & \\
\hline \multirow{4}{*}{ Abdomen } & \multirow{2}{*}{ No } & $\mathrm{N}$ & 446 & 140 & 84 & 76 & 746 & \multirow{4}{*}{0,000} \\
\hline & & $\%$ & 59,79 & 18,77 & 11,26 & 10,19 & 100 & \\
\hline & \multirow{2}{*}{ Sí } & $\mathrm{N}$ & 34 & 27 & 46 & 58 & 165 & \\
\hline & & $\%$ & 20,61 & 16,36 & 27,88 & 35,15 & 100 & \\
\hline \multirow{4}{*}{ Miembros superiores } & \multirow{2}{*}{ No } & $\mathrm{N}$ & 288 & 83 & 80 & 80 & 531 & \multirow{4}{*}{0,097} \\
\hline & & $\%$ & 54,24 & 15,63 & 15,07 & 15,07 & 100 & \\
\hline & \multirow{2}{*}{ Sí } & $\mathrm{N}$ & 192 & 84 & 50 & 54 & 380 & \\
\hline & & $\%$ & 50,53 & 22,11 & 13,16 & 14,21 & 100 & \\
\hline & $\mathrm{No}$ & $\mathrm{N}$ & 210 & 73 & 52 & 66 & 401 & \\
\hline Miembros inferiores & 100 & $\%$ & 52,37 & 18,20 & 12,97 & 16,46 & 100 & 0501 \\
\hline 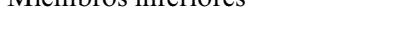 & Sí & $\mathrm{N}$ & 270 & 94 & 78 & 68 & 510 & o,jol \\
\hline & $\mathrm{Ni}$ & $\%$ & 52,94 & 18,43 & 15,29 & 13,33 & 100 & \\
\hline & No & $\mathrm{N}$ & 455 & 162 & 123 & 123 & 863 & \\
\hline Ouemado & & $\%$ & 52,72 & 18,77 & 14,25 & 14,25 & 100 & 0255 \\
\hline 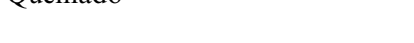 & Sí & $\mathrm{N}$ & 25 & 5 & 7 & 11 & 48 & (2) \\
\hline & $\mathrm{Ni}$ & $\%$ & 52,08 & 10,42 & 14,58 & 22,92 & 100 & \\
\hline Total & & $\mathrm{N}$ & 480 & 167 & 130 & 134 & 911 & \\
\hline IVtal & & $\%$ & 52,69 & 18,33 & 14,27 & 14,71 & 100 & \\
\hline
\end{tabular}

N: Número de pacientes. *Chi cuadrado de Pearson.

Fuente: Elaboración propia. 
Tabla 5. Asociación entre la puntuación del mISS y el número de áreas anatómicas afectadas.

\begin{tabular}{|c|c|c|c|c|c|c|c|c|c|c|}
\hline \multirow{2}{*}{$\begin{array}{c}\text { NÚMERO DE ÁREAS } \\
\text { ANATÓMICAS AFECTADAS }\end{array}$} & \multicolumn{10}{|c|}{ PUNTUACIÓN DEL mISS } \\
\hline & $\mathbf{N}$ & $\%$ & Media & DE & Mediana & P25 & P75 & Mínimo & Máximo & $\mathbf{p}^{*}$ \\
\hline 1 & 441 & 48,41 & 8,47 & 8,16 & 5 & 4 & 10 & 1 & 50 & \multirow{7}{*}{0,000} \\
\hline 2 & 254 & 27,88 & 11,97 & 10,92 & 8 & 4 & 17 & 1 & 50 & \\
\hline 3 & 147 & 16,14 & 14,74 & 12,98 & 9 & 5 & 22 & 1 & 57 & \\
\hline 4 & 51 & 5,60 & 20,51 & 13,49 & 19 & 9 & 30 & 2 & 57 & \\
\hline 5 & 13 & 1,43 & 23,46 & 10,05 & 22 & 19 & 25 & 11 & 50 & \\
\hline 6 & 5 & 0,55 & 29,60 & 22,19 & 41 & 6 & 48 & 5 & 48 & \\
\hline Total & 911 & 100,00 & 11,46 & 10,98 & 8 & 4 & 16 & 1 & 57 & \\
\hline
\end{tabular}

$\mathrm{N}$ : Número de pacientes. DE: Desviación estándar. P25: Percentil 25. P75: Percentil 75. *Kruskal Wallis.

Fuente: Elaboración propia.

Tabla 6. Asociación entre el nivel de gravedad del mISS y el número de áreas anatómicas afectadas.

\begin{tabular}{|c|c|c|c|c|c|c|c|}
\hline \multirow{2}{*}{\multicolumn{2}{|c|}{$\begin{array}{c}\text { NÚMERO DE ÁREAS } \\
\text { ANATÓMICAS AFECTADAS }\end{array}$}} & \multicolumn{6}{|c|}{ NIVEL DE GRAVEDAD mISS } \\
\hline & & Menor & Moderado & Grave & Crítico & Total & $\mathbf{p}^{*}$ \\
\hline \multirow{2}{*}{1} & $\mathrm{~N}$ & 264 & 88 & 55 & 34 & 441 & \multirow{14}{*}{0,000} \\
\hline & $\%$ & 59,86 & 19,95 & 12,47 & 7,71 & 100 & \\
\hline \multirow{2}{*}{2} & $\mathrm{~N}$ & 134 & 41 & 38 & 41 & 254 & \\
\hline & $\%$ & 52,76 & 16,14 & 14,96 & 16,14 & 100 & \\
\hline \multirow{2}{*}{3} & $\mathrm{~N}$ & 68 & 25 & 21 & 33 & 147 & \\
\hline & $\%$ & 46,26 & 17,01 & 14,29 & 22,45 & 100 & \\
\hline \multirow{2}{*}{4} & $\mathrm{~N}$ & 12 & 11 & 10 & 18 & 51 & \\
\hline & $\%$ & 23,53 & 21,57 & 19,61 & 35,29 & 100 & \\
\hline \multirow{2}{*}{5} & $\mathrm{~N}$ & 0 & 2 & 6 & 5 & 13 & \\
\hline & $\%$ & 0,00 & 15,38 & 46,15 & 38,46 & 100 & \\
\hline \multirow{2}{*}{6} & $\mathrm{~N}$ & 2 & 0 & 0 & 3 & 5 & \\
\hline & $\%$ & 40,00 & 0,00 & 0,00 & 60,00 & 100 & \\
\hline \multirow{2}{*}{ Total } & $\mathrm{N}$ & 480 & 167 & 130 & 134 & 911 & \\
\hline & $\%$ & 52,69 & 18,33 & 14,27 & 14,71 & 100 & \\
\hline
\end{tabular}

N: Número de pacientes. *Chi cuadrado de la razón de verosimilitud.

Fuente: Elaboración propia.

\section{DISCUSIÓN}

Los conflictos ocurridos en Afganistán e Irak, ambos en los que España participó, han constituido el mayor despliegue de tropas en operaciones militares en el presente siglo, con un coste de más de 22.000 y 35.000 bajas de combate estadounidenses, respectivamente ${ }^{7}$.

En Role 2 español de Herat se atendieron a 911 bajas de combate que sufrieron un total de 1.689 lesiones en donde las extremidades fueron la principal región afectada por heridas de guerra. De forma análoga, nuestros resultados coinciden con los datos obtenidos en la mayoría de las series publicadas ${ }^{8-17}$, en donde las extremidades, y en particular los miembros inferiores, fueron las áreas anatómicas más comúnmente lesionadas en las bajas de combate. Este predominio de lesiones en los miembros pudiera deberse, entre otros factores, al mayor porcentaje de superficie corporal que suponen las extremidades respecto al resto de regiones del cuerpo $\mathrm{y}$, a la utilización por parte de determinados combatientes, de medidas de protección pasiva (casco balístico y chaleco antifragmentos) sobre la cabeza, el tórax y la región abdominopélvica. Pese a ello, dos investigaciones recientes han encontrado que se ha producido una disminución estadísticamente significativa en el porcentaje de lesiones en las extremidades en las bajas de combate durante las operaciones «Iraqi Freedom»(OIF) y «Enduring Freedom» (OEF) con respecto a los datos obtenidos de la Segunda Guerra Mundial, y las guerras de Corea y Vietnam ${ }^{11,18}$.

Como se ha podido observar, la mayoría de las lesiones en bajas de combate se producen en los tejidos blandos de las extremidades. En contrapartida, estudios basados en los resultados de las autopsias realizadas a fallecidos estadounidenses durante la Segunda Guerra Mundial y la Guerra de Vietnam, y a fallecidos israelíes en el Líbano, han demostrado que la mayoría de las muertes se debieron a lesiones en la cabeza y en el tórax ${ }^{19,20}$.

Stevenson et al. han estudiado a todas las bajas militares británicas con lesiones por arma de fuego entre los años 2003 y 2014, en donde las regiones corporales más afectadas fueron las extremidades, con un $52 \%$, seguido por un $16 \%$ de lesiones por arma de fuego en la cabeza, un $15 \%$ en el tórax y un $7 \%$ en el abdomen ${ }^{21}$. En nuestra serie el grupo de 316 bajas de combate heridas por arma de fuego presentaron 429 lesiones de guerra, siendo las «extremidades inferiores» $(38,46 \%)$ la región corporal más afectada, seguido por las «extremidades superiores» $(25,87 \%)$ y la región de «tórax-dorso» $(13,51 \%)$. Al igual 
que en nuestro trabajo, en el estudio de Ramasamy et al. las bajas por heridas por arma de fuego presentaron un porcentaje significativamente mayor de lesiones en el tórax y en el abdomen en comparación con las bajas por explosivos ${ }^{22}$.

En la literatura reciente, la incidencia de lesiones de combate en las regiones de la «cabeza» y el «cuello» oscila entre el 13 y el $45 \%$ del total de lesiones sufridas por el personal combatiente, lo que hace que estas lesiones sean las segundas más comunes por detrás de las lesiones en las extremidades. Este aumento en la incidencia de lesiones en la cabeza y el cuello pudiera atribuirse a tres razones principales, el aumento en la supervivencia de la baja de combate, el uso habitual de explosivos que provocan un aumento de lesiones por múltiples fragmentos, y la falta de protección adecuada para la cara y el cuello ${ }^{23}$.

Conviene destacar que en aquellos conflictos que se desarrollan en entornos urbanos o semiurbanos, el patrón de lesión de la baja de combate puede verse modificado, como ocurrió por ejemplo durante la batalla de Mogadiscio (Somalia) en el año 1993, en donde las lesiones penetrantes fatales, de las 125 bajas de combate estadounidenses registradas, se distribuyeron del siguiente modo: heridas en la cabeza y la cara (36\%), cuello $(7 \%)$, tórax $(14 \%)$, abdomen $(14 \%)$, toracoabdominales $(7 \%)$, pelvis (14\%), y las extremidades $(7 \%)^{24}$.

La distribución anatómica de las heridas de guerra en la baja de combate puede verse modificada si observamos conflictos desarrollados en distintos TO, aunque las extremidades parecen continuar siendo la región corporal más frecuentemente afectada en los distintos conflictos armados recientes y actuales ${ }^{25-28}$.

Pese a que la cabeza, la cara y el cuello suponen únicamente el $12 \%$ del área total de la superficie corporal expuesta durante el combate ${ }^{29}$, estas áreas anatómicas sufren desproporcionadamente mayor número de lesiones y mayor número de lesiones potencialmente letales que el resto de regiones corporales. Un estudio durante Operation Iraqi Freedom OIF en el año 2004 analizó a 441 bajas estadounidenses con lesiones en cabeza, cara y cuello. La mediana del ISS fue de 2, y las puntuaciones medias del MaxAIS fueron de 2,1 $\pm 1,3$ para la cabeza; $1,1 \pm 0,3$ para la cara y; 1,2 $\pm 0,7$ para el cuello ${ }^{30}$. En el Role 2 español de Herat, la mediana de las puntuaciones del mISS en las bajas que presentaron heridas en la región de cabeza/cara y en el cuello fue de 8 y 5, respectivamente. Estas puntuaciones más elevadas pudieran deberse al empleo de la versión militar de la escala AIS, la cual otorga puntuaciones más elevadas a este tipo de lesiones.

También se ha estudiado que la presencia de cualquier fractura facial se encuentra significativamente asociada con una puntuación mayor en el índice de gravedad de las bajas ${ }^{31}$. Y más recientemente, en un trabajo realizado por Breeze et al. sobre bajas de combate con lesiones penetrantes en el cuello, se ha visto que la mortalidad en estas bajas se encuentra asociada de forma positiva con la puntuación del ISS en el momento de la llegada de la baja una formación sanitaria ${ }^{32}$.

Dentro del grupo de lesiones en la cabeza, conviene destacar las lesiones del oído, como uno de los tipos de lesión más frecuentemente encontrado en las bajas de combate que sufren lesiones por onda de blast $^{33,34}$. Su prevalencia se estima entre el $24 \%$ y el $40 \%$ en las series de bajas por explosivos en atentados terroristas ${ }^{35-38}$, sin embargo en la literatura militar encontramos resultados dispares, desde un 5\% de las lesiones en bajas de combate británicas ${ }^{39}$, hasta estudios en donde las lesiones del oído fueron las más frecuentes, correspondiendo a un $23 \%$ de las lesiones sufridas por las bajas de un batallón de infantería mecanizada en Irak ${ }^{40}$. Más concretamente, la perforación de la membrana timpánica es una de las tres lesiones que se han descrito como patognomónicas del mecanismo primario de blast injury ${ }^{41}$, con una incidencia en torno al $15 \%$ en bajas de combate heridas por explosivos ${ }^{42,43}$, y su presencia se encuentra relacionada con el desarrollo de cuadros de conmoción cerebral ${ }^{44}$, pérdida de capacidad auditiva ${ }^{42}$ y tinnitus ${ }^{45}$.

Además, en una revisión retrospectiva realizada sobre 23 atentados terroristas con bajas masivas acontecidos en Israel entre los años 1994 y 2005, la presencia de una perforación de la membrana timpánica se asoció con un aumento de la puntuación del ISS y del número de áreas anatómicas lesionadas. También se asoció con la necesidad de tratamiento quirúrgico, con una mayor estancia hospitalaria, con la admisión del paciente en la UCI, y con una mayor necesidad de traslado a un centro de trauma de nivel $1^{46}$.

Por otro lado, se ha sugerido que las rupturas de la membrana timpánica inducidas por explosión poseen un valor pronóstico predictivo del $50 \%$ de lesión pulmonar concomitante ${ }^{47,48}$, aunque este hallazgo ha sido debatido en estudios militares recientes en donde el hallazgo de una perforación de la membrana timpánica presentó una baja sensibilidad para la predicción de otras lesiones primarias por onda de blast $^{43}$, o expresado de otro modo, la presencia de una membrana timpánica intacta en bajas lesionadas por explosivos no excluye de la presencia de otras lesiones primarias $^{43}$ ni pulmonares ${ }^{49}$ por onda de blast.

La región abdominal ha sido una de las áreas anatómicas menos afectadas en las bajas de combate procedentes de los conflictos de Irak y Afganistán. No obstante, las lesiones penetrantes en el abdomen se relacionaron con puntuaciones elevadas del NISS entre las bajas de combate atendidas en el Role 3 británico de Camp Bastion (Afganistán) entre el mes de junio de 2006 y el mes de junio de $2013^{50}$. Al igual que las 977 bajas de combate con lesiones colorrectales procedentes de OIF y de Operation Eduring Freedom (OEF) entre los años 2003 y 2011, en las que la puntuación media del ISS fue de 22,2, con unas tasas elevadas de mortalidad, incluso después de ser atendidas en una formación sanitaria con capacidad de Role $2^{51}$.

En nuestra serie, 165 (18,11\%) bajas habían sufrido lesiones abdominales. Estas lesiones constituyeron la región anatómica con la puntuación más alta respecto del resto de áreas corporales. Además, la distribución de las puntuaciones del mISS fue estadísticamente diferente entre las bajas que sufrieron lesiones en el abdomen y las que no las sufrieron. Más aún, de las 165 bajas con lesiones abdominales más de dos tercios de los sujetos fueron categorizados con un nivel de gravedad grave o crítico (puntuación > 15). Estos datos reflejan la gravedad de las lesiones abdominales en el entorno militar, motivo por el cual los oficiales médicos especialistas en Cirugía General son un elemento imprescindible entre el personal que conforma una formación sanitaria de tipo Role 2, que es aquella que cuenta con capacidad de proporcionar elementos de reanimación y cirugía de control del daño, y de tratamiento y hospitalización de bajas ${ }^{52}$.

Algo similar ocurre con las heridas de guerra que afectan al tórax, pese a su no tan elevada frecuencia, las 1.660 bajas de 


\section{El patrón de lesión y su relación con el índice de gravedad en la baja de combate}

combate con lesiones torácicas procedentes de OIF y OEF entre los años 2002 y 2008, presentaron una mortalidad del 12,1\% y una puntuación media del ISS de 14,953. En otro estudio, desde enero de 2003 hasta mayo de 2011, se registraron 23.797 bajas de combate estadounidenses procedentes de OEF y OIF, 2.048 de las cuales sufrieron alguna lesión torácica, lo que supone una prevalencia del 8,6\%, con una puntuación media del ISS de 22,6 . Un $35,5 \%$ de las bajas con lesiones torácicas presentaron una puntuación del ISS menor de 16, entre 16 y 24 en el $27 \%$ de las bajas, y superior a 25 en el $37.5^{\%} \%^{54}$. En nuestro trabajo, $201(22,01 \%)$ bajas presentaron lesiones de combate en la región del tórax/dorso. Al igual que en las bajas con lesiones abdominales, la distribución de las puntuaciones del mISS fue estadísticamente significativa entre los sujetos que sufrieron lesiones en el tórax/dorso y los que no las sufrieron. Y más de un tercio de las bajas con lesiones torácicas fueron categorizadas con un nivel de gravedad crítico (puntuaciones 25-75).

En cuanto a las lesiones torácicas no penetrantes en la baja de combate, la lesión de estructuras mediastínicas se ha considerado como un factor fuertemente relacionado con la mortalidad, pudiendo llegar a considerar estas lesiones como potencialmente letales ${ }^{55}$.

Las hemorragias en el tronco y en las zonas de unión de las extremidades con el tronco han sido una de las causas de muerte más frecuente en bajas de combate con lesiones potencialmente no letales ${ }^{56-59}$. Varios autores sugieren que la baja incidencia de lesiones torácicas durante OEF y OIF pudiera deberse a un uso extendido entre los combatientes de chalecos de protección balística, a sus mejoras en la cobertura de su área de superficie y a la mejora de la capacidad de la armadura para desviar los proyectiles de alta velocidad ${ }^{8,60}$. Otros autores además sugieren que el uso de los chalecos de protección balística ha contribuido a disminuir la incidencia de lesiones abdominales ${ }^{24,61,62}$. Se considera que las mejoras tecnológicas en los chalecos de protección balística han contribuido al aumento observado en la supervivencia de las bajas de combate desde Vietnam. Los chalecos antifragmentos comenzaron a tener un uso generalizado durante la Operación «Desert Storm» («Tormenta del Desierto»), y su uso se ha ido extendiendo progresivamente durante los conflictos recientes. En Vietnam, la tasa de lesiones torácicas fue del $13 \%$, mientras que en OEF y OIF, esta tasa disminuyó a un $5 \%{ }^{8}$. Asimismo, en un análisis de 4.700 bajas de combate se observó que la tasa de lesiones torácicas fue del $18 \%$ en aquellos individuos que no llevaban chalecos de protección balística, y menor al $5 \%$ en aquellos que si usaron esta medida de protección pasiva ${ }^{60}$.

Lakstein et al. han estudiado el patrón de lesión en 96 bajas del ejército israelí durante la intifada de Al-Aqsa. Entre las bajas que fueron categorizadas como ISS menor (puntuación 1-8) la mayoría de las heridas de guerra afectaron a las extremidades $(56,3 \%)$, cabeza, cuello y cara $(33,3 \%)$ y tronco $(14,6 \%)$; entre las categorizada como ISS moderado (puntuación 9-15) un treinta por ciento de las bajas presentaron lesiones en la cabeza, el cuello y la cara, y un veinte por ciento sufrieron heridas en el tronco. Curiosamente, todas las bajas clasificadas con un nivel de gravedad moderado resultaron heridas en una sola región corporal. Cinco bajas presentaron una puntuación ISS superior a 15 , tres $(60 \%)$ sufrieron heridas de por arma de fuego en el tronco y dos $(40 \%)$ sufrieron quemaduras extensas y heridas en el tronco y las extremidades como resultado de una explosión ${ }^{63}$. En nuestra serie, la distribución de los niveles de gravedad del mISS en función del patrón lesional mostró que las heridas de guerra en los miembros inferiores fueron la lesión predominante en todos los grupos. Es decir, en las bajas de combate atendidas en el Role 2 español de Herat la lesión de los miembros inferiores fue el patrón de lesión más frecuentemente encontrado independientemente del nivel de gravedad en el que fueron clasificadas.

Por último, en los últimos años algunos autores han tratado de evaluar que factores influyen en la puntuación del ISS. En un estudio en el que se analizaron 153 bajas militares tailandesas encontraron que los factores que influyeron en el ISS con una diferencia estadísticamente significativa fueron la edad, la lesión abdominal, la lesión de cabeza y cuello, y la lesión torácica $^{64}$. Por otra parte, otros autores han tratado de estudiar los índices de gravedad como predictores de infección en lesiones de combate. En el estudio de Penn-Barwell et al. utilizaron el New Injury Severity Score (NISS) ${ }^{65}$, cuyo cálculo se realiza mediante la suma del cuadrado de las tres lesiones con puntuación más alta independientemente de la región anatómica afectada, para categorizar a 89 bajas de combates con fracturas abiertas de tibia que habían sido tratadas en el Role 4 británico en un periodo de diez años, y al igual que otros autores ${ }^{66}$, descubrieron que la gravedad de la lesión no estaba asociada con el riesgo de infección ${ }^{67}$.

\section{CONCLUSIONES}

El patrón de lesión en las bajas de combate atendidas en el Role 2 español de Herat, tanto en heridos por arma fuego como por explosivos, se caracterizó por la predominante afectación de las extremidades, principalmente de los miembros inferiores. La distribución anatómica de las heridas de guerra encontrada en los pacientes de nuestro estudio se asemeja a los resultados publicados en otras series que han analizado el patrón de lesión en bajas de combate procedentes de los conflictos de Irak y Afganistán.

Por otra parte, los resultados de este trabajo sugieren que las heridas de guerra que afectan al tórax/dorso o al abdomen podrían considerarse como un factor predictor del aumento de la gravedad la baja de combate. Además, hemos podido constatar la existencia una relación directa entre la gravedad y el número de áreas anatómicas afectadas en este tipo de paciente.

No obstante, y a pesar de la alta prevalencia de lesiones en múltiples regiones corporales, hasta la fecha ningún estudio ha evaluado el significado de las distintas combinaciones específicas de regiones anatómicas lesionadas en la baja de combate. Son necesarios más trabajos en esta línea para permitirnos una mejor comprensión de la relación existente entre el patrón de lesión y la gravedad en la baja de combate.

\section{BIBLIOGRAFÍA}

1. NATO Standardization Office (NSO). AAP-6. NATO Glossary of terms and definitions. 2019.

2. Atlas of injuries in the U.S. Armed Forces. Mil Med. 1999;164(8 Suppl):S1-89.

3. Champion HR, Bellamy RF, Roberts CP, Leppaniemi A. A Profile of Combat Injury. J Trauma. 2003; 54(5): 13-9. 
4. Baker SP, O'Neill B, Haddon W, Long WB. The injury severity score: a method for describing patients with multiple injuries and evaluating emergency care. J Trauma. 1974; 14(3): 187-96.

5. Champion HR, Holcomb JB, Lawnick MM, Kelliher T, Spott MA, Galarneau MR, et al. Improved characterization of combat injury. J Trauma. 2010; 68(5): 1139-50.

6. Bolorunduro OB, Villegas C, Oyetunji TA, Haut ER, Stevens KA, Chang DC, et al. Validating the injury severity score (ISS) in different populations: ISS predicts mortality better among hispanics and females. J Surg Res. 2011; 166(1): 40-4.

7. Blum DA, DeBruyne NF. American war and military operations casualties: Lists and statistics. En: Key Congressional Reports for September 2019: Part IX. 2020.

8. Owens BD, Kragh JF, Wenke JC, Macaitis J, Wade CE, Holcomb JB. Combat wounds in operation Iraqi Freedom and operation Enduring Freedom. J Trauma. 2008; 64(2): 295-9.

9. Shen-Gunther J, Ellison R, Kuhens C, Roach CJ, Jarrard S. Operation Enduring Freedom: Trends in Combat Casualty Care by Forward Surgical Teams Deployed to Afghanistan. Mil Med. 2011; 176(1): 67-78.

10. Ramasamy A, Harrison S, Lasrado I, Stewart M. A review of casualties during the Iraqi insurgency 2006 - A British field hospital experience. Injury. 2009; 40(5): 493-7.

11. Belmont PJ, Goodman GP, Zacchilli M, Posner M, Evans C, Owens BD Incidence and epidemiology of combat injuries sustained during «the surge» portion of operation Iraqi Freedom by a U.S. Army brigade combat team. J Trauma. 2010; 68(1): 204-10.

12. Hoencamp R, Idenburg FJ, Hamming JF, Tan ECTH. Incidence and epidemiology of casualties treated at the dutch role 2 enhanced medical treatment facility at multi national base Tarin Kowt, Afghanistan in the period 20062010. World J Surg. 2014; 38(7): 1713-8.

13. Poyat C, Boutonnet M, de Saint Maurice GP, Ausset S, Daban J-L, Blot R-M. Profile of wounded French war fighters in Afghanistan: Experience of the French military medical service over the period 2001-2010. Anesth Reanim. 2017; 3(5): 403-12.

14. Schoenfeld AJ, Dunn JC, Belmont PJ. Pelvic, spinal and extremity wounds among combat-specific personnel serving in Iraq and Afghanistan (2003-2011): A new paradigm in military musculoskeletal medicine. Injury. 2013; 44(12): 1866-70.

15. Belmont PJ, McCriskin BJ, Sieg RN, Burks R, Schoenfeld AJ. Combat wounds in Iraq and Afghanistan from 2005 to 2009. J Trauma Acute Care Surg. 2012; 73(1): 3-12.

16. Schoenfeld AJ, Dunn JC, Bader JO, Belmont PJ. The nature and extent of war injuries sustained by combat specialty personnel killed and wounded in Afghanistan and Iraq, 2003-2011. J Trauma Acute Care Surg. 2013; 75(2): 287-91.

17. Janak JC, Mazuchowski EL, Kotwal RS, Stockinger ZT, Howard JT, Butler FK, et al. Patterns of Anatomic Injury in Critically Injured Combat Casualties: A Network Analysis. Sci Rep. 2019; 9(13767): 1-10

18. Owens BD, Kragh JF, Macaitis J, Svoboda SJ, Wenke JC. Characterization of extremity wounds in operation Iraqi freedom and operation enduring freedom. J Orthop Trauma. 2007; 21(4): 254-7.

19. Gofrit ON, Kovalski N, Leibovici D, Shemer J, O'Hana A, Shapira SC. Accurate anatomical location of war injuries: Analysis of the Lebanon war fatal casualties and the proposition of new principles for the design of military personal armour system. Injury. 1996; 27(8): 577-81.

20. Garfield R, Neugut A. Epidemiologic analysis of warfare. A historical review. JAMA Surg. 1991; 266(5): 688-92.

21. Stevenson T, Carr DJ, Penn-Barwell JG, Ringrose TJ, Stapley SA. The burden of gunshot wounding of UK military personnel in Iraq and Afghanistan from 2003-14. Injury. 2018; 49(6): 1064-9.

22. Ramasamy A, Harrisson SE, Clasper JC, Stewart MPM. Injuries from roadside improvised explosive devices. J Trauma. 2008; 65(4): 910-4.

23. Tong D, Beirne R. Combat Body Armor and Injuries to the Head, Face, and Neck Region: A Systematic Review. Mil Med. 2013; 178(4): 421-6.

24. Mabry RL, Holcomb JB, Baker AM, Cloonan CC, Uhorchak JM, Perkins DE, et al. United States army rangers in Somalia: An analysis of combat casualties on an urban battlefield. J Trauma. 2000; 49(3): 515-29.

25. Bodalal Z, Mansor S. Gunshot injuries in Benghazi-Libya in 2011: The Libyan conflict and beyond. Surgeon. 2013; 11(5): 258-63.
26. Dubost C, Goudard Y, Soucanye de Landevoisin E, Contargyris C, Evans D, Pauleau G. Combat casualties from two current conflicts with the Seventh French Forward Surgical Team in Mali and Central African Republic in 2014. J R Army Med Corps. 2016; 162(6): 450-5.

27. Barbier O, Malgras B, Versier G, Pons F, Rigal S, Ollat D. French surgical experience in the Role 3 Medical Treatment Facility of KaIA (Kabul International Airport, Afghanistan): The place of the orthopedic surgery. Orthop Traumatol Surg Res. 2014; 100(6): 681-5.

28. Hussain N, Okeke IBJ, Oyebanji AE, Akunne JI, Omoruyi OJ. Combat injuries sustained by troops on counter terrorism and counter-insurgency operations in North east Nigeria: Implications for intervention. African J Emerg Med. 2020

29. Carey ME. Learning From Traditional Combat Mortality and Morbidity Data Used in the Evaluation of Combat Medical Care. Mil Med. 1987; 152(1): 6-13.

30. Wade AL, Dye JL, Mohrle CR, Galarneau MR. Head, face, and neck injuries during operation Iraqi Freedom II: Results from the Us Navy-Marine Corps Combat Trauma Registry. J Trauma. 2007; 63(4): 836-40.

31. Wordsworth M, Thomas R, Breeze J, Evriviades D, Baden J, Hettiaratchy S. The surgical management of facial trauma in British soldiers during combat operations in Afghanistan. Injury. 2017; 48(1): 70-4.

32. Breeze J, Bowley DM, Combes JG, Baden J, Orr L, Beggs A, et al. Outcomes following penetrating neck injury during the Iraq and Afghanistan conflicts: A comparison of treatment at US and United Kingdom medical treatment facilities. J Trauma Acute Care Surg. 2020; 88(5): 696-703.

33. Chandler DW. Blast-Related Ear Injury in Current U.S. Military Operations. ASHA Lead. 2006; 11(9): 8-29.

34. Guzman Rosario D, Noguera Marín M, Santillán Sánchez M, Manjarrés Henríquez F, Cid Bello M, Navarro Suay R. Incidencia del barotrauma ótico en bajas de combate por artefacto explosivo improvisado. Póster presentado en: I Congreso de Sanidad Militar. Granada, España. 2014.

35. Mrena R, Pääkkönen R, Bäck L, Pirvola U, Ylikoski J. Otologic consequences of blast exposure: A finnish case study of a shopping mail bomb explosion. Acta Otolaryngol. 2004; 124(8): 946-52.

36. Tungsinmunkong S, Chongkolwatana C, Piyawongvisal W, Atipas S, Namchareonchaisuk S. Blast injury of the ears: The experience from Yala Hospital, Southern Thailand. J Med Assoc Thail. 2007; 90(12): 2662-8.

37. Walsh RM, Pracy JP, Huggon AM, Gleeson MJ. Bomb blast injuries to the ear: The London Bridge incident series. J Accid Emerg Med. 1995; 12(3): 194-8.

38. Persaud R, Hajioff D, Wareing M, Chevretton E. Otological trauma resulting from the Soho Nail Bomb in London, April 1999. Clin Otolaryngol Allied Sci. 2003; 28(3): 203-6.

39. Breeze J, Cooper H, Pearson CR, Henney S, Reid A. Ear injuries sustained by British service personnel subjected to blast trauma. J Laryngol Otol. 2011; 125(1): 13-7.

40. Gondusky JS, Reiter MP. Protecting Military Convoys in Iraq: An Examination of Battle Injuries Sustained by a Mechanized Battalion during Operation Iraqi Freedom II. Mil Med. 2005; 170(6): 546-9.

41. DePalma RG, Burris DG, Champion HR, Hodgson MJ. Blast Injuries. N Engl J Med. 2005; 352(13): 1335-42.

42. Ritenour AE, Wickley A, Ritenour JS, Kriete BR, Blackbourne LH, Holcomb JB, et al. Tympanic Membrane Perforation and Hearing Loss From Blast Overpressure in Operation Enduring Freedom and Operation Iraqi Freedom Wounded. J Trauma. 2008; 64(2): 174-8.

43. Harrison CD, Bebarta VS, Grant GA. Tympanic Membrane Perforation After Combat Blast Exposure in Iraq: A Poor Biomarker of Primary Blast Injury. J Trauma. 2009; 67(1): 210-1.

44. Xydakis MS, Bebarta VS, Harrison CD, Conner JC, Grant GA, Robbins AS Tympanic-membrane perforation as a marker of concussive brain injury in Iraq. N Engl J Med. 2007; 357(8): 830-1.

45. Dougherty AL, MacGregor AJ, Han PP, Viirre E, Heltemes KJ, Galarneau MR. Blast-related ear injuries among U.S. military personnel. J Rehabil Res Dev. 2013; 50(6): 893-904.

46. Ashkenazi I, Olsha O, Turegano-Fuéntes F, Alfici R. Tympanic membrane perforation impact on severity of injury and resource use in victims of explosion. Eur J Trauma Emerg Surg. 2017; 43(5): 623-6.

47. Almogy G, Luna T, Richter E, Pizov R, Bdolah-Abram T, Mintz Y, et al. Can external signs of trauma guide management? Lessons learned from suicide bombing attacks in Israel. Arch Surg. 2005; 140(4): 390-3. 


\section{El patrón de lesión y su relación con el índice de gravedad en la baja de combate}

48. Ciraulo DL, Frykberg ER. The Surgeon and Acts of Civilian Terrorism: Blast Injuries. J Am Coll Surg. 2006; 203(6): 942-50.

49. Peters P. Primary Blast Injury: An Intact Tympanic Membrane Does Not Indicate the Lack of a Pulmonary Blast Injury. Mil Med. 2011; 176(1): 110-4.

50. Leong MJ, Edgar I, Terry M. Penetrating abdominal injury: UK military experience from the Afghanistan conflict. J R Nav Med Serv. 2016; 102(2): 90-4.

51. Glasgow SC, Steele SR, Duncan JE, Rasmussen TE. Epidemiology of modern battlefield colorectal trauma: A review of 977 coalition casualties. J Trauma Acute Care Surg. 2012; 73(6): 503-8.

52. NATO Standardization Office (NSO). STANAG 2598. AJMedP-8, Edition A, Version 1. Allied Joint Medical Doctrine Military Health Care. 2018.

53. Propper BW, Gifford SM, Calhoon JH, McNeil JD. Wartime Thoracic Injury: Perspectives in Modern Warfare. Ann Thorac Surg. 2010; 89(4): 1032-6.

54. Ivey KM, White CE, Wallum TE, Aden JK, Cannon JW, Chung KK, et al Thoracic injuries in US combat casualties: A 10-year review of Operation Enduring Freedom and Iraqi Freedom. J Trauma Acute Care Surg. 2012, 74(6): 514-9.

55. Pearce AP, Bull AMJ, Clasper JC. Mediastinal injury is the strongest predictor of mortality in mounted blast amongst UK deployed forces. Injury. 2017; 48(9): 1900-5.

56. Eastridge BJ, Hardin M, Cantrell J, Oetjen-Gerdes L, Zubko T, Mallak C, et al. Died of wounds on the battlefield: Causation and implications for improving combat casualty care. J Trauma. 2011; 71(1): 4-8.

57. Morrison JJ, Oh J, Dubose JJ, O'Reilly DJ, Russell RJ, Blackbourne LH, et al. En-route care capability from point of injury impacts mortality after severe wartime injury. Ann Surg. 2013; 257(2): 330-4.

58. Kelly JF, Ritenour AE, McLaughlin DF, Bagg KA, Apodaca AN, Mallak $\mathrm{CT}$, et al. Injury severity and causes of death from Operation Iraqi Freedom and Operation Enduring Freedom: 2003-2004 versus 2006. J Trauma. 2008; 64(2): 21-7.

59. Holcomb JB, McMullin NR, Pearse L, Caruso J, Wade CE, Oetjen-Gerdes L, et al. Causes of death in U.S. special operations forces in the global war on terrorism: 2001-2004. Ann Surg. 2007; 245(6): 986-91.

60. Eastridge BJ, Jenkins D, Flaherty S, Schiller H, Holcomb JB. Trauma system development in a theater of war: Experiences from operation Iraqi freedom and operation enduring freedom. J Trauma. 2006; 61(6): 1366-73.

61. Zouris JM, Walker GJ, Dye J, Galarneau M. Wounding Patterns for U.S Marines and Sailors during Operation Iraqi Freedom, Major Combat Phase. Mil Med. 2006; 171(3): 246-52.

62. Chambers LW, Rhee P, Baker BC, Perciballi J, Cubano M, Compeggie M, et al. Initial experience of US Marine Corps forward resuscitative surgical system during operation Iraqi freedom. Arch Surg. 2005; 140(1): 26-32.

63. Lakstein D, Blumenfeld A. Israeli Army Casualties in the Second Palestinian Uprising. Mil Med. 2005; 170(5): 427-30

64. Boonthep N, Intharachat S, Iemsomboon T. Factors influencing injury severity score regarding Thai military personnel injured in mass casualty incident April 10, 2010: Lessons learned from armed conflict casualties: A retrospective study. BMC Emerg Med. 2012; 12(1): 1-7.

65. Osler T, Baker SP, Long W. A modification of the injury severity score that both improves accuracy and simplifies scoring. J Trauma. 1997; 43(6): 922-6.

66. Dellinger EP, Miller SD, Wertz MJ, Grypma M, Droppert B, Anderson PA. Risk of Infection After Open Fracture of the Arm or Leg. Arch Surg. 1988; 123(11): 1320-7.

67. Penn-Barwell JG, Bennett PM, Mortiboy DE, Fries CA, Groom AFG, Sargeant ID. Factors influencing infection in 10 years of battlefield open tibia fractures. Strateg Trauma Limb Reconstr. 2016; 11(1): 13-8. 